Journal of Bangladesh Academy of Sciences, Vol. 37, No. 2, 139-144, 2013

\title{
ENERGY RESOLUTION AND GAS GAIN OF AN ACTIVE-TARGET TYPE GAS COUNTER OF GEM-MSTPC
}

\author{
SURANJAN KUMAR DAS ${ }^{*}$, HIRONOBU ISHIYAMA ${ }^{1}$, YUTAKA WATANABE ${ }^{1}$, \\ HIROARI MIYATAKE ${ }^{1}$, YOSHIKAZU HIRAYAMA ${ }^{1}$, SUNCHAN JEONG ${ }^{1}$ AND \\ KANAKO YAMAGUCHI ${ }^{2}$
}

Department of Physics, Jagannath University, Dhaka-1100, Bangladesh

\begin{abstract}
An investigation was made on the energy resolution of an active-target type gas counter operating in low-pressure $\mathrm{He} / \mathrm{CO}_{2}$ mixed gas for studies of nuclear reactions such as $(\alpha, n)$ and $(\alpha, p)$ using radioactive nuclear beams (RNBs). The dependencies of effective gain and energy resolution on different parameters such as drift field, induction field and GEM voltages were studied. The energy resolution with adequate electric field configuretaion was found to be $3 \%$ in $\sigma$.
\end{abstract}

Key words: THGEM, GEM, Energy resolution, Drift field, Induction field, GEM voltage

\section{INTRODUCTION}

Multi-sampling and Tracking Proportional Chamber with Gas Electron Multiplier (GEM-MSTPC, Das et al. 2011, Yamaguchi et al. 2010) configured with thick GEM (THGEM), is an active target type gas counter operating with low-pressure $\mathrm{He} / \mathrm{CO}_{2}$ mixed gas for studies of nuclear reactions such as $(\alpha, n)$ and $(\alpha, p)$ using radioactive nuclear beams (RNBs). For identification of a reaction event, dE/dx method i.e., the energy loss per unit length is used. In this process the energy $(\mathrm{dE} / \mathrm{dx})$ resolution of the counter should be better for clear selection of the reaction event. The energy resolution of GEM-MSTPC was around $8 \%$ in $\sigma$ for ${ }^{12} \mathrm{C}$ beam (Ishiyama et al. 2012). This value was sufficient for identification of the reaction event induced by a heavy ion beam with the mass number of around 10. For further studies of reactions induced by heavy-ion beams having the mass number of around 40, relatively high energy resolution is required.

The energy resolution has been investigated at an off-line test bench using $\alpha$ rays fed from an Am- $\alpha$ source. In order to simulate the energy resolution of heavier ions by $\alpha$ rays, experimental conditions such as gas pressure and counter geometry were chosen. As for example to measure ${ }^{44} \mathrm{Ti}(\alpha, \mathrm{p})^{47} \mathrm{~V}$ reaction, which is considered to be one of key reactions in supernovae nucleosynthesis, the energy loss per anode pad (length $=4.2 \mathrm{~mm}$ )

* Corresponding author: <skdas252002@yahoo.com>.

${ }^{1}$ Institute of Particle and Nuclear studies, High Energy Accelerator Research Organization (KEK), 1-1 Oho, Tsukuba, Ibaraki 305-0801, Japan.

${ }^{2}$ University of Tsukuba, 1-1-1 Tsukuba, Ibaraki 305-8571, Japan. 
is $1.3 \mathrm{MeV}$ for ${ }^{44} \mathrm{Ti}$ at the gas pressure of 120 torr in GEM-MSTPC. In this case, the required values of resolution and effective gas gain are less than $3 \%$ in $\sigma$ and about 30 40 , respectively. In order to simulate the energy loss of ${ }^{44} \mathrm{Ti}$ in GEM-MSTPC by $\alpha$ particle, a long anode pad (length $=96 \mathrm{~mm}$, not segmented) was used. The number of seed electrons of ${ }^{44} \mathrm{Ti}$ at short anode pad at 120 torr is equivalent to the one of $\alpha$ particle at long anode pad at 320 torr. In order to investigate a correlation between energy resolution and the energy loss (i.e. the number of seed electrons), different gas pressures from 120 to 420 torr, covering the condition for the measurement of ${ }^{44} \mathrm{Ti}(\alpha, \mathrm{p}){ }^{47} \mathrm{~V}$ reaction were used in this study. The estimated values of energy loss, using SRIM code (www.srim.org), are $0.45,0.97,1.59$ and $1.97 \mathrm{MeV}$ in 120, 240, 360 and 420 torr, respectively, for $\mathrm{He} / \mathrm{CO}_{2}(90 \% / 10 \%)$ mixed gas. In those selected gas pressures, dependencies of effective gain and energy resolution on different parameters such as drift field, induction field and GEM voltages were investigated. In the following sections, the detail of the methods and the results with discussion are presented.

\section{MATERIALS AND METHODS}

A thick GEM (THGEM) used in this study was similar reported by Das et al. (2011) and Yamaguchi et al. (2010). The THGEM was fabricated on a $400 \mu \mathrm{m}$-thick print circuit board (PCB) clad on both sides with an 8 - $\mu \mathrm{m}$ thick $\mathrm{Cu}$ layer coated by a $1 \mu \mathrm{m}$-thick gold and had an FR4 based insulator. The hole diameter and pitch were $500 \mu \mathrm{m}$ and $700 \mu \mathrm{m}$, respectively. After the holes were pierced with a drill, the board was washed with $3 \%$ hydrochloric acid $(\mathrm{HCl})$.

The structure of GEM-MSTPC and the experimental setup are shown in Fig.1. Detailed structure of GEM-MSTPC has been already reported by Ishiyama et al. (2012). The detector consists of a field cage, GEM and a readout anode. The field cage has a cubic volume $100 \mathrm{~cm}^{3}$. A uniform electric field was applied in vertical direction in the field cage. One or more THGEMs are mounted between the drift space and the segmented anode plate. In this study, single GEM configuration was selected for simplicity. Electrons produced by the ionization of charged particles drift toward the anode plate through the GEMs. The anode plate below the GEM is segmented into 24 pads. Each pad with a length of $4.2 \mathrm{~mm}$ along to the beam injection axis consists of two triangular electrodes to measure not only energy losses of charged particles but also those positions in the horizontal plane by charge division. In this study, the long anode plate with a length of $96 \mathrm{~mm}$ was used instead of segmented anode pad as mentioned before. The vertical position of the particles can be obtained from the electron drift time. The induction gap between GEM and the anode plate was $2 \mathrm{~mm}$. The height of the drift space was $100 \mathrm{~mm}$ usually. In this study, in order to cover a wide range of drift field, the height was set at $10 \mathrm{~mm}$ as shown in Fig.1. 
The counter gas was $\mathrm{He} / \mathrm{CO}_{2}(90 \% / 10 \%)$, which was operated at a pressure of 120 , 240, 360 and 420 torr. An ${ }^{241} \mathrm{Am}-\alpha$ source with a collimator hole had diameter of $6 \mathrm{~mm}$. In order to generate a trigger signal, a Si solid-state detector (SSD) with a $10 \times 10 \mathrm{~mm}^{2}$ active area was installed opposite to the $\alpha$ source. The distance between the Am $\square$ source and SSD was $220 \mathrm{~mm}$. The anode signal was read out by a charge sensitive preamplifier. The amplifier had a conversion gain of $1 \mathrm{~V} / \mathrm{pC}$ and a reset time-constant of $0.5 \mu \mathrm{s}$; it was followed by a amplifier (ORTEC 570). Output signals and noise level from the amplifier were monitored and recorded by an oscilloscope and/or a multi-channel analizer through an analog-to-degital converter. During the measurements of the resolution, we paid attentions to the following points, such as the white noise on base line of output signal from a pre-amplifier, the stability for electric voltage sources, and the stability of gas pressure.

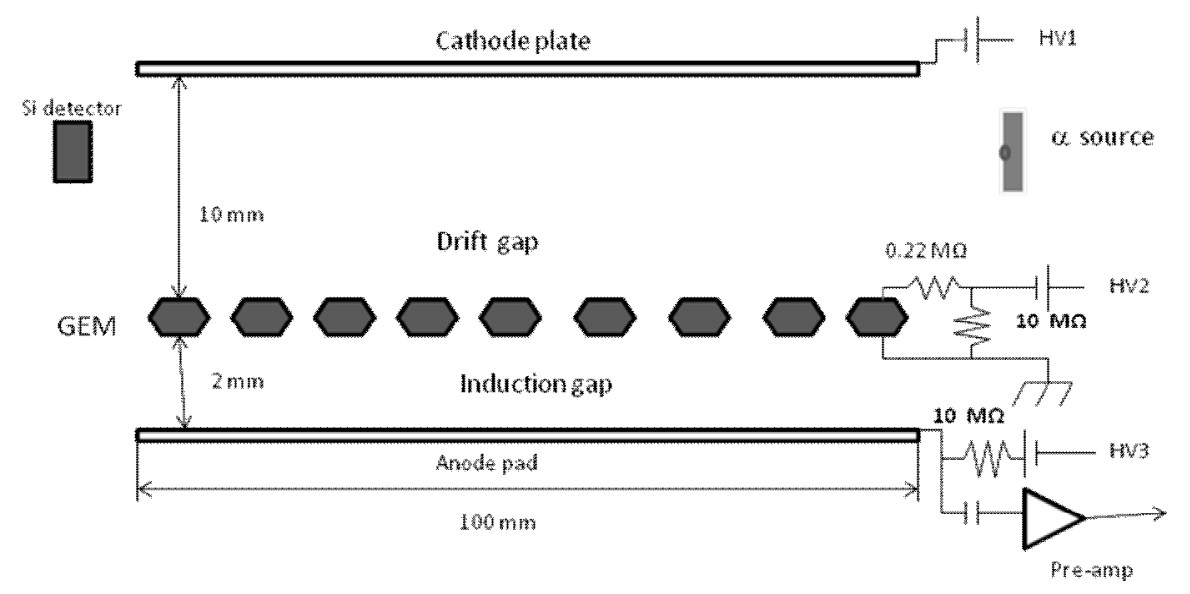

Fig.1. Schematic cross sectional view of GEM-MSTPC with the experimental setup used for this study.

\section{RESULTS AND DISCUSSION}

Electrons produced by an incident $\alpha$ particle drift towards the GEM and are multiplied as they pass through the holes of the GEM. A part of these electrons drift towards the anode and are readout by the preamplifier, and some are absorbed in an electrode of THGEM. The readout electrons are a fraction of the total number of electrons produced by the GEM; hence, the effective gain ' $G$ ' is evaluated instead of absolute gas gain, by using the formula, $\mathrm{G}=\mathrm{N}_{\mathrm{e}} / \mathrm{N}_{\text {seed }}$, where $\mathrm{N}_{\mathrm{e}}$ is the number of electrons evaluated from the pulse height of the output pulse from the preamplifier with the conversion gain of $1 \mathrm{~V} / \mathrm{pC}$, and $\mathrm{N}_{\text {seed }}$ is the number of seed electrons estimated from initial charge generated by the ionization process induced by an $\alpha$ particle: energy loss of an $\alpha$ particle passing through the active area is calculated using SRIM code and the 
number of electron-ion pairs created by the energy loss process is estimated. The energy resolution was obtained from the distribution of output signals from the anode.

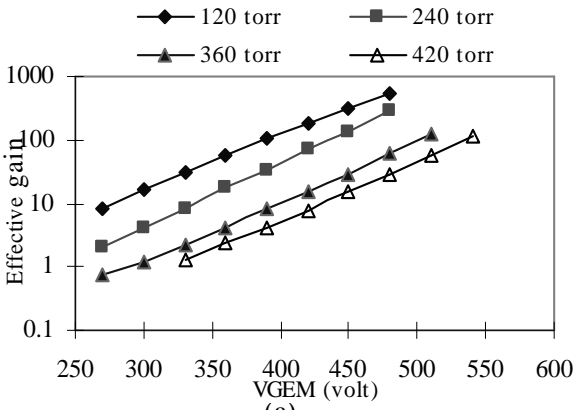

(a)

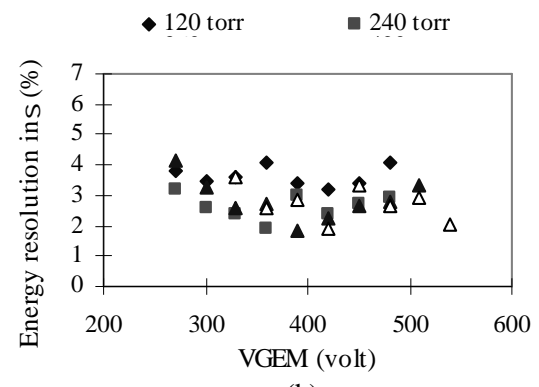

(b)

Fig.2. (a) Effective gas gain as a function of $\mathrm{V}_{\mathrm{GEM}}$ at various gas pressures from 120 to 420 torr; (b) Energy resolution as a function of $\mathrm{V}_{\mathrm{GEM}}$ at various gas pressures from 120 to 420 torr.

The voltage applied to electrodes of the GEM (GEM voltage, $\mathrm{V}_{\mathrm{GEM}}$ ) forms a strong electric field inside the holes, which leads to the gas multiplication process. Fig. 2(a) shows the effective gas gain as a function of $\mathrm{V}_{\mathrm{GEM}}$. The drift field and the induction field during these measurements were typically set at $1.0 \mathrm{kV} / \mathrm{cm}$.atm and $4.7 \mathrm{kV} / \mathrm{cm}$.atm, respectively (Ishiyama et al. (2012). The gain increased with increased $\mathrm{V}_{\mathrm{GEM}}$. This was because the avalanche electrons gradually increased as the voltage increases. Figure 2(b) shows the energy resolution as a function of $\mathrm{V}_{\mathrm{GEM}}$ at various gas pressured. Energy resolutions were stable with $\mathrm{V}_{\mathrm{GEM}}$. The results show a little variation with the pressure, i.e., energy resolution does not depend on gas pressure within measured range.

The dependencies of effective gain and resolution upon the drift field were examined. Fig. 3(a) shows the effective gain as a function of drift field, where the induction field was set at $4.7 \mathrm{kV} / \mathrm{cm}$.atm. The values of $\mathrm{V}_{\mathrm{GEM}}$ were selected 330,385 , 450 and $480 \mathrm{~V}$ for 120, 240, 360 and 420 torr, respectively in order to obtain required gas gain of around $30-40$ for ${ }^{44} \mathrm{Ti}(\alpha, \mathrm{p})$ measurement at typical drift field of $1.0 \mathrm{kV} / \mathrm{cm}$. atm and induction field of $4.7 \mathrm{kV} / \mathrm{cm}$.atm. As shown in the figure, effective gain increases with drift field as it is below some specific value and after this value it decreases. At the relatively high drift field, some field lines terminate on the upper electrode of the GEM, so that only a certain fraction of the drifting electrons is collected in the holes. As a result the effective gain decreases with increasing drift field. On the other hand, diffusion increases with decreasing drift field. An increase of diffusion causes a loss of electrons. So, the effective gain is smaller at lower drift field (Bouclier et al. (1997)). Figure 3(b) shows the energy resolution as a function of drift field. The energy resolution is large at low drift field because the diffusion effect reduces the number of primary electrons. As drift field increases up to a specific value, the transmission of electrons increases with 
drift field and better energy resolution is obtained. After some specific value of the drift field, the electron transmission reduces due to field line termination on the upper electrode of GEM. In this case the energy resolution should be increased. But the experimental values of energy resolution were found nearly stable. So, further investigation is necessary.

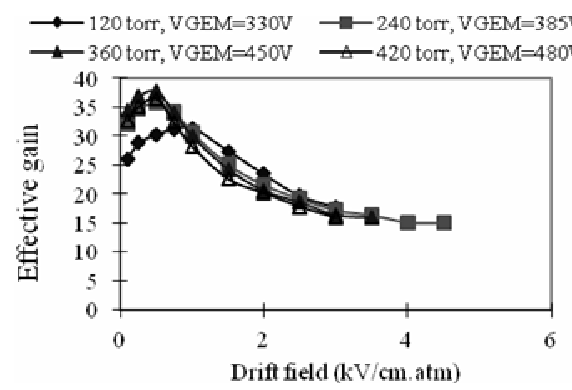

(a)

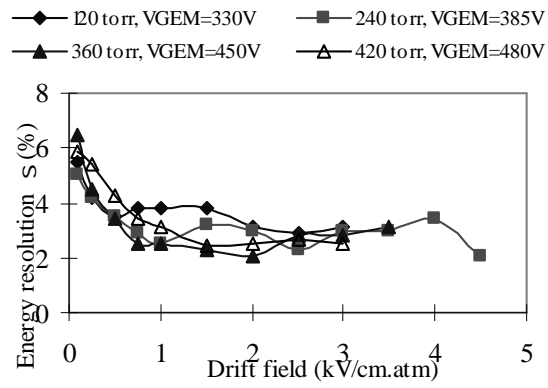

(b)

Fig. 3. (a) Effective gain as a function of drift field; (b) Energy resolution as a function of drift field.

Induction field: The fraction of electrons extracted from the GEM holes into the induction gap depends on the induction field. Fig.4 (a) shows the effective gain as a function of induction field. The drift field was set at $1.0 \mathrm{kV} / \mathrm{cm}$.atm and $\mathrm{V}_{\mathrm{GEM}}$ were selected same values in the measurement for drift field. As shown in the figure, effective gain increases slowly with induction field. This is due to loss of extraction electrons in the induction region at the lower induction field. Parallel plate multiplication (PPAC mode) begins in the induction region above $6 \mathrm{kV} / \mathrm{cm}$.atm Das et al. (2011).

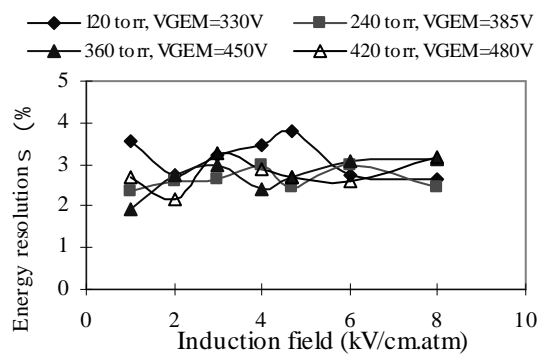

(a)

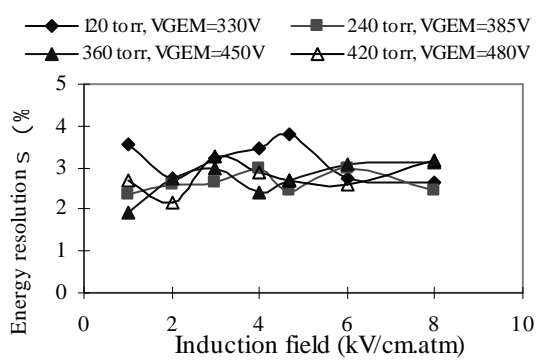

(b)

Fig. 4. (a) Effective gain as a function of induction field; (b) Energy resolution as a function of induction field.

This mode is considered unsafe, because it may allow a discharge in GEM to propagate to readout electrode. Fig. 4 (b) shows the energy resolution as a function of induction field. As shown in the figure, the energy resolution does not depend on the induction field. 


\section{CONCLUSION}

Gas gain and energy resolution were measured at various conditions. Results showed that gas gain increased with $\mathrm{V}_{\mathrm{GEM}}$. Energy resolution depended little on $\mathrm{V}_{\mathrm{GEM}}$. The energy resolution was stable at the drift fields above $1.0 \mathrm{kV} / \mathrm{cm}$.atm. Further investigation is necessary to explain this behavior. In case of induction field, effective gain increased slowly with increasing induction field. The energy resolution was stable all over the measured range of induction field. As the result indicates, if the drift field is set above $1 \mathrm{kV}$ $/ \mathrm{cm}$.atm with adequate $\mathrm{V}_{\mathrm{GEM}}$ and induction field, the energy resolution of around $3 \%$ in $\sigma$ can be achieved. Therefore, this detector can be applicable for the studies of reactions induced by the heavy-ion beams having the mass number of around 40 .

\section{ACKNOWLEDGEMENT}

Authors are grateful to radioactive nuclear beam R\&D group of KEK for their assistance and providing with test instruments as well as their laboratory space.

\section{REFERENCES}

Bouclier, R., W. Dominik, M. Hoch, J. C. Labbe, G. Million, L. Ropelewski, F. Sauli, A. Sharma and G. Manzin. 1997. New Observations with the gas electron multiplier (GEM). Nucl. Instrum. Methods A 396: 50-66.

Das, S.K., Y. Mizoi, T. Fukuda, K. Yamaguchi, H. Ishiyama, M.H. Tanaka, Y.X. Watanabe and H. Miyatake. 2011. Gas gain study of standard CERN GEM and $400 \mathrm{~mm}$-Thick GEM in lowpressure $\mathrm{He} / \mathrm{CO}_{2}$ mixed gas, Nucl. Instrum. Methods A 625: 39-42.

Ishiyama, H., K. Yamaguchi, Y. Mizoi, Y.X. Watanabe, S.K. Das, T. Hashimoto, H. Miyatake, Y. Hirayama, N. Imai, M. Oyaizu, S.C. Jeong, T. Fukuda, S. Mitsuoka, H. Makii and T.K. Sato. 2012. GEM-MSTPC: An active-target type detector in low-pressure $\mathrm{He} / \mathrm{CO}_{2}$ mixed gas, JINST 7 C03036 (http://iopscience.iop.org/1748-0221/7/03/C03036):1-14.

Yamaguchi K., H. Ishiyama, M.H. Tanaka, Y.X. Watanabe, H. Miyatake, Y. Hirayama, N. Imai, H. Makki, Y. Fuchi, S.C. Jeong,. T. Nomura, Y. Mizoi, S.K. Das, T. Fukuda, T. Hashimoto and I. Arai. 2010. Development of GEM-MSTPC for measurements of lowenergy nuclear reactions, Nucl. Instrum. Methods A 623: 135-137. 